\title{
Malondialdehyde-Modified Low-Density Lipoprotein Is a Useful Marker to Identify Patients With Vulnerable Plaque
}

Kenichiro Tajika, MD; Kentaro Okamatsu, MD; Masamichi Takano, MD; Shigenobu Inami, MD; Masanori Yamamoto, MD; Daisuke Murakami, MD; Nobuaki Kobayashi, MD; Takayoshi Ohba, MD; Noritake Hata, MD; Yoshihiko Seino, MD; Kyoichi Mizuno, MD

Background: The association between elevated malondialdehyde-modified low-density lipoprotein (MDA-LDL) and plaque instability in patients with coronary artery disease (CAD) is suspected but not established. The aim of the present study was therefore to investigate the association between serum MDA-LDL and plaque characteristics on angioscopy.

Methods and Results: A total of 37 consecutive patients with CAD and single-vessel disease who underwent preinterventional angioscopy, were studied. Using angioscopy at the target lesions, the presence of yellow plaque and complex plaque was examined. Moreover, we evaluated the yellow intensity, which has been shown to have an inverse correlation with the fibrous-cap thickness of the plaques, with quantitative colorimetry to identify a thin-cap atheroma. Serum MDA-LDL in patients with thin-cap atheroma diagnosed on quantitative colorimetry was significantly higher than in patients without thin-cap atheroma $(\mathrm{P}<0.0009)$. Univariate logistic regression indicated that serum MDA-LDL was a predictor for thin-cap atheroma (odds ratio [OR], 1.48; 95\% confidence interval [Cl]: 1.101.97; $\mathrm{P}=0.003)$ and for complex plaque (OR, 1.22; 95\% $\mathrm{Cl}: 1.00-1.48 ; \mathrm{P}=0.046)$. On multivariate logistic regression serum MDA-LDL was the only independent predictor for thin-cap atheroma (OR, 1.48; 95\% Cl: 1.10-1.97; $\mathrm{P}=0.011)$.

Conclusions: Using angioscopy and quantitative colorimetry, elevated MDA-LDL was confirmed to be associated with thin-cap atheroma in CAD patients. (Circ $J$ 2012; 76: 2211-2217)

Key Words: Angioscopy; Lipoprotein; Plaque vulnerability

$\mathbf{O}$ xidative modification of low-density lipoprotein cholesterol (LDL-C) is believed to play a key role in the initiation and progression of atherosclerosis. ${ }^{1-3}$ In particular, elevated malondialdehyde-modified LDL (MDA-LDL), one of the oxidized forms, has been reported to be detected in the plasma of patients with acute coronary syndrome (ACS) but not in those with stable coronary artery disease (CAD) ${ }^{4,5}$ From these studies, it is reasonable to hypothesize that elevated MDALDL is associated with endothelial injury and/or plaque instability rather than with the extent of atherosclerosis, but this hypothesis has not yet been directly verified.

Coronary angioscopy can provide direct images of the endoluminal surface at lesions and enable us to detect the plaque instability by the evaluation of yellow plaque intensity and plaque complexity such as superimposed thrombus and intimal flap. ${ }^{6-8}$ Previously, the yellow plaque intensity was shown to have an inverse relationship with the overlying fibrous cap thick- ness, ${ }^{9}$ and quantitative evaluation of the yellow plaque intensity was reported to be available with newly developed software using $\mathrm{L}^{*} \mathrm{a} * \mathrm{~b} *$ color space, in which yellow intensity is represented as $b^{*} .{ }^{10}$ Angioscopy of ex vivo human tissue samples has indicated that a plaque with highly intense yellow color (b* $>23$ ) contained atheroma that had a fibrous cap thickness $<100 \mu \mathrm{m},{ }^{10}$ and the clinical relevance of this parameter has been suggested by use of the quantitative colorimetry system during angioscopy. ${ }^{11-13}$ Highly intense yellow plaque (HIYP) with $b^{*}$ $>23$ has been demonstrated to be strongly associated with disruption and/or coronary thrombosis in ACS patients, ${ }^{11}$ and with thin-cap fibroatheroma diagnosed on virtual histology intravascular ultrasound. ${ }^{13}$ In the present study, therefore, we investigated the relationship between circulating MDA-LDL and angioscopic plaque characteristics with quantitative colorimetry to verify the hypothesis that elevated serum MDA-LDL reflects plaque instability in patients with CAD.

Received February 10, 2012; revised manuscript received April 27, 2012; accepted May 18, 2012; released online June 19, 2012 Time for primary review: 26 days

Division of Cardiology, Nippon Medical School, Tokyo (K.T., K.O., S.I., K.M.); Cardiovascular Center, Nippon Medical School, ChibaHokusoh Hospital, Chiba (K.T., K.O., M.T., M.Y., D.M., N.K., T.O., N.H., Y.S.), Japan

Mailing address: Kentaro Okamatsu, MD, PhD, Cardiovascular Center, Nippon Medical School, Chiba Hokusoh Hospital, 1715 Kamakari, Inzai, Chiba 270-1694, Japan. E-mail: okamatsu@nms.ac.jp

ISSN-1346-9843 doi:10.1253/circj.CJ-12-0183

All rights are reserved to the Japanese Circulation Society. For permissions, please e-mail: cj@j-circ.or.jp 


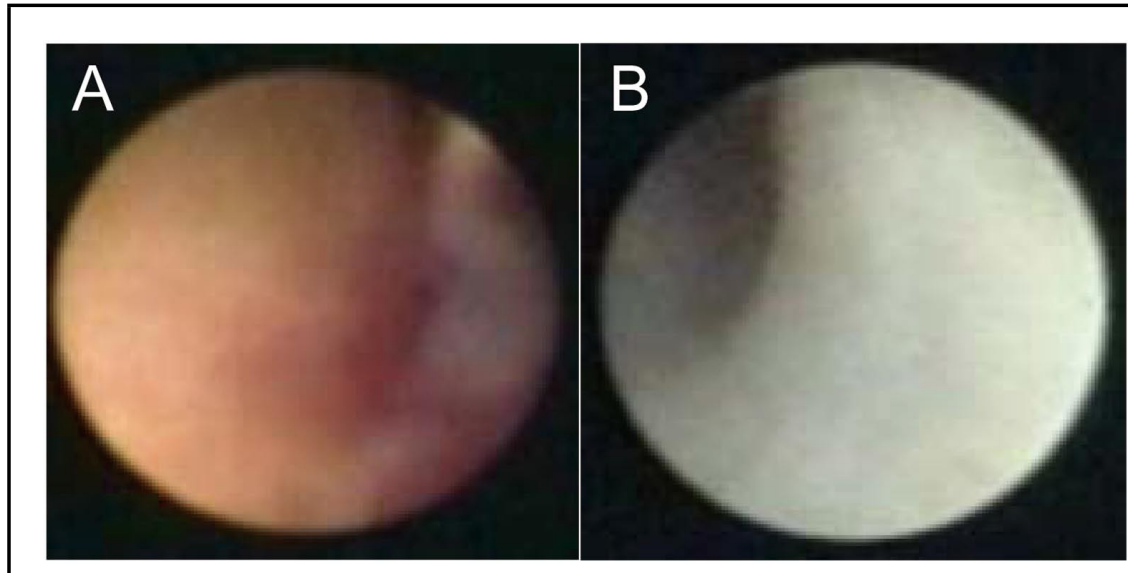

Figure 1. Angioscopic images. (A) Complex lesion with highly intense yellow plaque $\left(b^{*}=27.6\right)$ and mixed (red and white) thrombi. (B) Simple lesion with nearly white plaque $\left(b^{*}=6.8\right)$.

\section{Patients and Blood Sampling}

The subjects consisted of 37 consecutive patients with CAD and single-vessel disease (10 with ACS and 27 with stable CAD) who underwent pre-interventional angioscopy at Chiba Hokusoh Hospital. Catheter procedures were performed within 3 days after admission. Quantitative coronary angiography was done using the CMS (MEDICS) system. A coronary stenosis was considered clinically significant if it was $>50 \%$ in diameter. Single-vessel disease was presumed when only 1 coronary artery demonstrated significant coronary stenosis. Patients with ACS had ischemic chest discomfort at rest with ST-segment elevation or depression of $>0.5 \mathrm{~mm}$ or T-wave inversion of $>1 \mathrm{~mm}$. In 8 patients, elevated cardiac troponin T $(\mathrm{cTnT})(>0.01 \mathrm{ng} / \mathrm{ml})$ was present at entry or in the samples taken at 6-8h after enrollment, indicating acute myocardial infarction (AMI). In 2 patients, no cTnT elevation was found, and these patients were classified as having unstable angina. Twenty-seven patients with angiographically documented CAD and no clinical signs of ischemia at rest within the previous month were considered to have stable CAD. The exclusion criteria included multi-vessel disease, left main disease, left ventricular ejection fraction $<40 \%$, coronary artery bypass graft or PTCA performed within the last 6 months, renal failure, serious intercurrent disease, and coronary arteries unsuitable for angioscopy, such as being severely tortuous, diffuse and narrow, or having ostial lesions. Written informed consent approved by the institutional review board was obtained from all patients before participation.

Venous blood samples from all patients were obtained on admission. MDA-LDL measurement was performed using a sandwich ELISA method that has been previously described; the combination of monoclonal antibody against MDA (ML25) with monoclonal antibody against apoB (AB16) has been shown to recognize MDA-LDL. ${ }^{14}$ Total cholesterol, high-density lipoprotein (HDL)-C, and triglycerides were measured using standard enzymatic methods. LDL-C was calculated with the Friedewald formula.

\section{Coronary Angioscopy}

The angioscopic procedure using an imaging catheter (Vecmova Neo; FiberTech, Chiba, Japan) has been previously reported..$^{15}$ After performing routine coronary angiography, we observed the target lesion on coronary angioscopy before catheter intervention. The angioscopic images were digitally recorded in the same manner as previously reported, ${ }^{10}$ to avoid modifications of the coloration. The video signal was converted from analog to digital (A/D) by a converter (ADVC-100; Canopus, Kobe, Japan), which was directly connected to both the S (Y/C) signal output of the CCD camera and the 6-pin IEEE1394 connector of a desktop computer (Macintosh Power Mac G4, Apple Japan, Tokyo, Japan).

\section{Definition and Analysis of Angioscopic Findings}

$\mathrm{L} * \mathrm{a} * \mathrm{~b} *$ color space is widely used to describe all the colors visible to the human eye (http://en.wikipedia.org/wiki/Lab_ color_space). In this color space, yellow color intensity can be represented as $b^{*}$ (yellow color intensity $0-100$ ). The $b^{*}$ of plaques expressed by custom software based on the L*a*b* color space was reported to be independent of such conditions as light intensity, the distance from the angioscope's lens to the object, and even the angle of the angioscope to the region of interest, and to have an inverse linear correlation with the fibrous cap thickness of the plaques. ${ }^{10}$ HIYP was defined as plaque with $b^{*}>23$, based on studies that have identified this cut-off as optimal for identification of such plaque instability as a fibrous cap thickness $<100 \mu \mathrm{m} .{ }^{10,11}$ The patients were divided into 2 groups according to $b^{*}$ : those with HIYP and those without HIYP.

The presence of thrombus was defined as superficial or protruding red or white mass, adherent to the vessel surface but clearly a separate structure that persisted despite flushing with a saline solution. A complex plaque was considered to be present when the surface of the lesion had a thrombus and/or an irregular appearance with ulcerations, flaps, or fissures (Figure 1).

Two investigators performed quantitative colorimetric analysis and qualitative angioscopic analysis separately and independently. The intraobserver agreement on the angioscopic images was calculated by having an observer repeat the measurement of $b^{*}$ and the assessment on the 37 images (presented in random order) after 1 week. The interobserver agreement on the angioscopic images was calculated by comparing the $\mathrm{b}^{*}$ and the assessment on the 37 images by the 2 observers. The intraobserver agreement for $b^{*}$ was $r=0.98$ for the $1 \mathrm{ob}-$ server and $\mathrm{r}=0.96$ for the other observer $(\mathrm{P}<0.0001$ for both). The interobserver agreement, analyzed using the mean of the 2 measurements by each observer, was $\mathrm{r}=0.91 \quad(\mathrm{P}<0.0001)$. The mean $b^{*}$ of the 4 analyses by the 2 observers was expressed as the quantified yellow intensity of plaques at the target lesions. The intra- and interobserver agreements for the other evaluated angioscopic item (complex plaque) were 97\%, and 


\begin{tabular}{|c|c|c|c|}
\hline & HIYP $(n=16)$ & Without HIYP (n=21) & $P$ value \\
\hline Age (years) & $60.6 \pm 7.4$ & $59.8 \pm 10.5$ & 0.78 \\
\hline Male & $15(94)$ & $19(90)$ & $>0.99$ \\
\hline \multicolumn{4}{|l|}{ Risk factor } \\
\hline Diabetes mellitus & $5(31)$ & $6(29)$ & 0.85 \\
\hline Hypertension & $6(38)$ & $13(61)$ & 0.14 \\
\hline Hyperlipidemia & $14(87)$ & $13(62)$ & 0.14 \\
\hline Smoking & $3(19)$ & $0(0)$ & $>0.99$ \\
\hline $\mathrm{BMI}\left(\mathrm{kg} / \mathrm{m}^{2}\right)$ & $25.5 \pm 2.8$ & $24.1 \pm 3.2$ & 0.07 \\
\hline \multicolumn{4}{|l|}{ Diagnosis } \\
\hline ACS & $8(50)$ & $2(10)$ & 0.01 \\
\hline AMI & $7(44)$ & $1(5)$ & \\
\hline Unstable angina & $1(6)$ & $1(5)$ & \\
\hline Stable angina & $8(50)$ & $19(90)$ & \\
\hline \multicolumn{4}{|l|}{ Location of target lesion } \\
\hline RCA & $5(31)$ & $3(14)$ & 0.45 \\
\hline LAD & $9(56)$ & $14(67)$ & \\
\hline LCx & $2(13)$ & $4(19)$ & \\
\hline \multicolumn{4}{|l|}{ Serum lipid } \\
\hline MDA-LDL (U/L) & $125.7 \pm 52.5$ & $77.5 \pm 26.7$ & 0.0009 \\
\hline Total cholesterol (mg/dl) & $192.0 \pm 32.3$ & $186.7 \pm 27.8$ & 0.60 \\
\hline HDL-C (mg/dl) & $44.1 \pm 14.0$ & $49.1 \pm 16.4$ & 0.35 \\
\hline Triglycerides (mg/dl) & $155.1 \pm 68.1$ & $144.5 \pm 70.6$ & 0.64 \\
\hline LDL-C (mg/dl) & $116.7 \pm 27.0$ & $108.7 \pm 23.6$ & 0.34 \\
\hline Statin use & $8(50)$ & $11(52)$ & $>0.99$ \\
\hline \multicolumn{4}{|l|}{ Angioscopy parameters } \\
\hline$b^{*}$ & $28.2 \pm 4.6$ & $9.4 \pm 5.5$ & - \\
\hline Complex plaque & $9(56)$ & $3(14)$ & 0.01 \\
\hline
\end{tabular}

Data given as mean $\pm \mathrm{SD}$ or $\mathrm{n}(\%)$.

ACS, acute coronary syndrome; AMI, acute myocardial infarction; BMI, body mass index; HDL-C, high-density lipoprotein cholesterol; HIYP, highly intense yellow plaque; LAD, left anterior descending artery; LCx, left circumflex artery; LDL-C, low-density lipoprotein cholesterol; MDA-LDL, malondialdehyde-modified LDL-C; RCA, right coronary artery.

\begin{tabular}{|c|c|c|c|c|c|c|}
\hline & \multicolumn{3}{|c|}{ Univariate analysis } & \multicolumn{3}{|c|}{ Multivariate analysis } \\
\hline & $P$ value & OR & $95 \% \mathrm{Cl}$ & $P$ value & OR & $95 \% \mathrm{Cl}$ \\
\hline & \multicolumn{6}{|c|}{ Predictors for HIYP } \\
\hline Serum MDA-LDL level ${ }^{\ddagger}$ & $0.003^{\dagger}$ & 1.48 & $1.10-1.97$ & $0.011^{\dagger}$ & 1.48 & $1.10-1.97$ \\
\hline Diagnosis of ACS & $0.012^{\dagger}$ & 9.50 & $1.64-55.00$ & 0.313 & 2.67 & $0.29-24.63$ \\
\hline Complex plaque & $0.010^{\dagger}$ & 7.71 & $1.60-37.14$ & 0.386 & 5.69 & $0.42-75.87$ \\
\hline \multicolumn{7}{|c|}{ Predictors for complex plaque } \\
\hline Serum MDA-LDL level ${ }^{\ddagger}$ & $0.046^{\dagger}$ & 1.22 & $1.00-1.48$ & 0.547 & 1.00 & $0.82-1.48$ \\
\hline Diagnosis of ACS & $0.001^{\dagger}$ & 23.00 & $3.51-150.51$ & $0.010^{\dagger}$ & 13.44 & $1.84-97.75$ \\
\hline HIYP & $0.010^{\dagger}$ & 7.71 & $1.60-37.14$ & 0.360 & 2.66 & $0.32-21.73$ \\
\hline
\end{tabular}

Variables with $P>0.05$ on univariate analysis are not presented. †Statistically significant; $¥ 10-\mathrm{U} / \mathrm{L}$ increments.

$\mathrm{Cl}$, confidence interval; OR, odds ratio. Other abbreviations as in Table 1.

$97 \%$, respectively. When any discordance was observed in these items between the 2 observers, a third investigator read the images and a consensus was thus obtained.

\section{Statistical Analysis}

Continuous variables are expressed as mean \pm SD. Comparisons between the 2 groups were done using the unpaired Student's t-test. Comparisons of categorical variables presented as numbers $(\%)$ were done with either the chi-squared test or
Fisher's exact test. Univariate logistic regression analysis was used to determine the factors associated with the angioscopic findings (HIYP and complex plaques), respectively. The variables included in the analysis were classic coronary risk factors (age, sex, hypertension, hyperlipidemia, diabetes mellitus, smoking, body mass index), diagnosis, serum levels of lipids including MDA-LDL, frequency of statin use and the other angioscopic findings. They were then selected for multivariate logistic regression when they achieved significance on univari- 


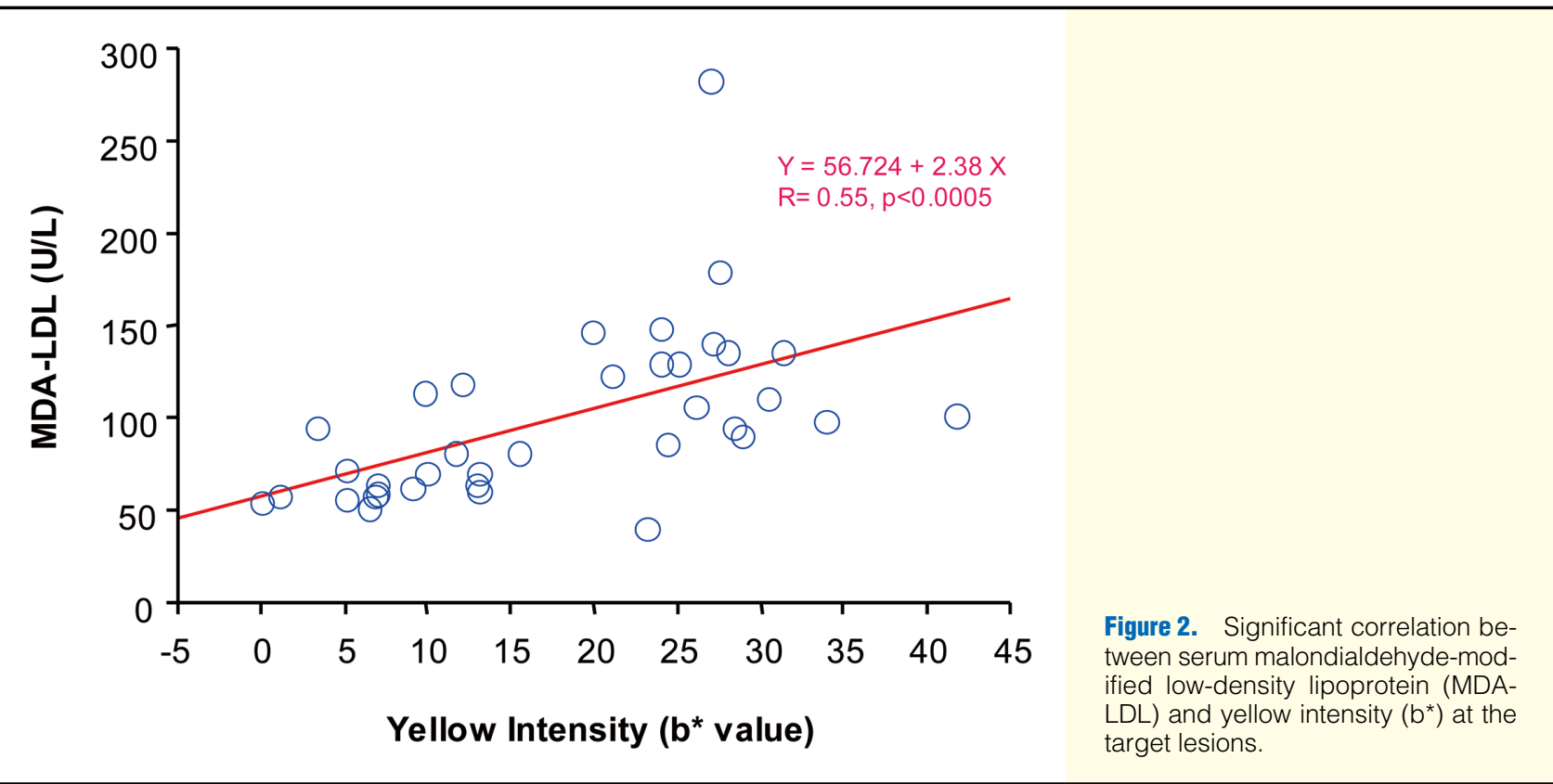

ate logistic regression analysis. The correlation between the 2 parameters was evaluated on linear regression analysis. We set the level of statistical significance at $\mathrm{P}<0.05$. For the identification of HIYP with different cut-offs of MDA-LDL, we computed the sensitivity, specificity, and accuracy, defined as the sum of the concordant cells divided by the sum of all cells in the $2 \times 2$ table. A receiver operating characteristic (ROC) curve was constructed to identify the optimal cut-off for MDA-LDL to detect HIYP. Using the optimal cut-off, we divided the patients into an elevated MDA-LDL group and a non-elevated MDA-LDL group.

Multivariate logistic regression analysis was used to determine the factors associated with elevated MDA-LDL in patients with CAD. Independent variables included in the analysis were the diagnosis of ACS and the angioscopic findings (HIYP and complex plaques), which were significant based on univariate logistic regression analysis.

\section{Results}

\section{Subjects}

Pre-interventional coronary angioscopy and successful percutaneous coronary intervention was performed in all patients without any serious procedural complications. The average of serum MDA-LDL in 37 patients was $98.3 \pm 46.1 \mathrm{U} / \mathrm{L}$. The clinical baseline characteristics and the lesion characteristics for the 2 groups are summarized in Table 1 . Serum MDA-LDL in the HIYP group was significantly higher than in the nonHIYP group (125.7 $\pm 52.5 \mathrm{U} / \mathrm{L}$ vs. 77.5 $\pm 26.7 \mathrm{U} / \mathrm{L}, \mathrm{P}<0.0009)$. The prevalence of ACS was higher in the HIYP group (50\% vs. $10 \%, \mathrm{P}=0.01$ ), and the frequency of angioscopic complex plaque was 4 -fold higher in the HIYP group (56\% vs. $14 \%$, $\mathrm{P}=0.01$ ). These complex plaques were accompanied with thrombus except in 1 patient. There were no significant differences in the frequency of risk factors for CAD and statin use or in the serum lipids except for MDA-LDL between the patients with and without HIYP.

\section{Predictors of Plaque Instability}

Univariate logistic regression analysis indicated that serum MDA-LDL level, complex plaques, and the diagnosis of ACS were predictors for HIYP. Multivariate logistic regression analysis showed serum MDA-LDL level to be the only independent predictor associated with HIYP (odds ratio [OR], 1.48; 95\% confidence interval (CI): 1.10-1.97; $\mathrm{P}=0.011$; Table 2). For complex plaques, univariate logistic analysis indicated that serum MDA-LDL level, HIYP, and diagnosis of ACS were predictors, and multivariate logistic analysis showed the diagnosis of ACS to be the only independent predictor (Table 2).

\section{MDA-LDL and Yellow Plaque}

Serum MDA-LDL level was significantly correlated with yellow intensity $\left(\mathrm{b}^{*}\right)$ at the target lesion plaques $(\mathrm{r}=0.55, \mathrm{P}<0.0005$; Figure 2). The serum levels of other lipids such as total cholesterol $(\mathrm{r}=0.11, \mathrm{P}<0.49)$, LDL-C $(\mathrm{r}=0.14, \mathrm{P}<0.39)$, HDL-C $(\mathrm{r}=-0.22, \mathrm{P}<0.18)$, and triglycerides $(\mathrm{r}=0.23, \mathrm{P}<0.16)$ were not correlated with yellow intensity, respectively.

The sensitivity and specificity for HIYP with regard to MDALDL were $88 \%$ and $76 \%$, respectively, when $90 \mathrm{U} / \mathrm{L}$ was used as the cut-off (Figure 3). ROC analysis showed an MDA-LDL level of 90U/L to be the optimal cut-off for presence of HIYP. The clinical characteristics and the lesion characteristics in patients with MDA-LDL $\geq 90 \mathrm{U} / \mathrm{L}(\mathrm{n}=18)$ and in those with MDALDL $<90 \mathrm{U} / \mathrm{L}(\mathrm{n}=19)$ are summarized in Table 3 . The patients with elevated serum MDA-LDL were more frequently observed to have ACS, HIYP, or complex plaques. Multivariate logistic regression analysis showed the presence of HIYP to be the only independent factor associated with elevated MDALDL in patients with CAD (OR, 21.8; 95\% CI: 3.14-152.06; $\mathrm{P}<0.002$; Table 4).

\section{Discussion}

This is the first study to show that increased MDA-LDL in patients with CAD is strongly associated with yellow plaques with a thin fibrous cap. This supports the hypothesis that elevated MDA-LDL reflects plaque instability in patients with 


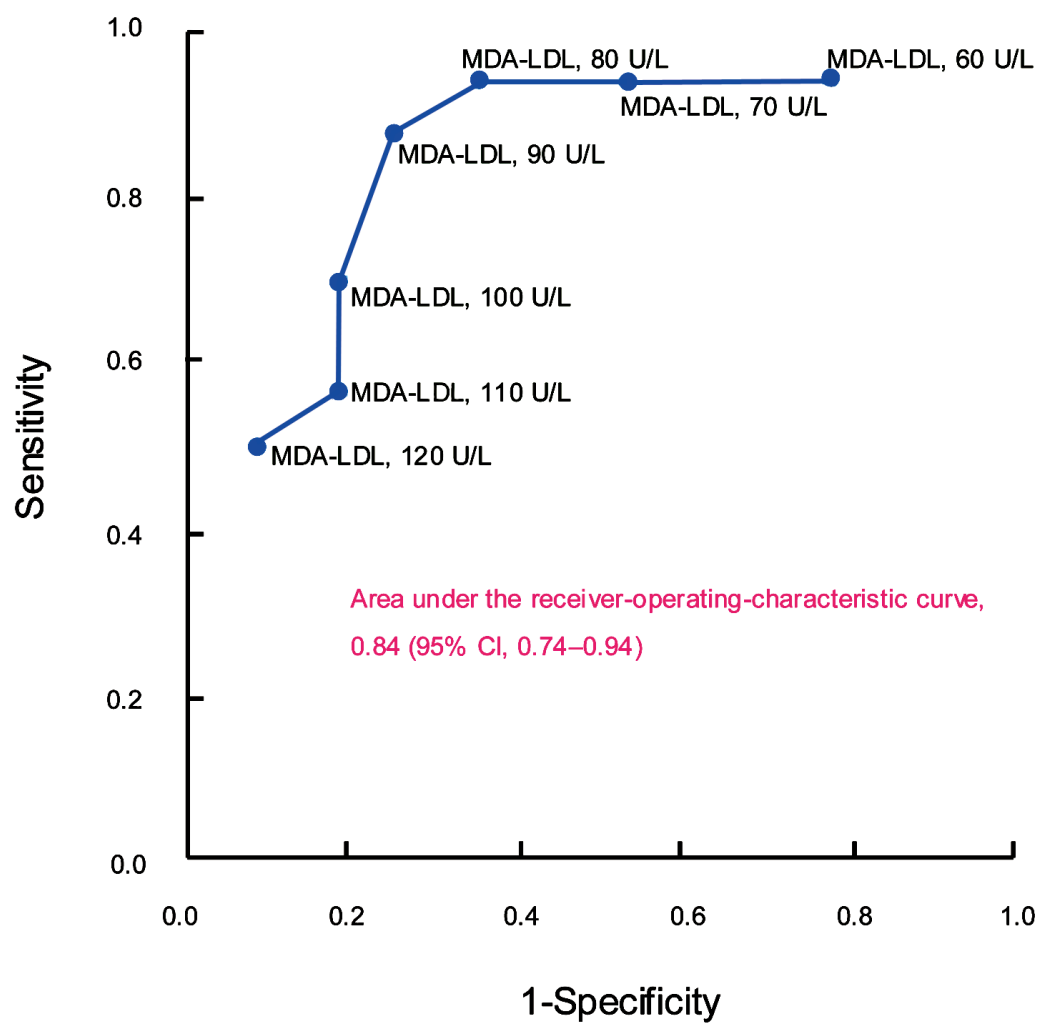

\begin{tabular}{cccccc}
$\begin{array}{c}\text { MDA-LDL } \\
\text { U/L }\end{array}$ & $\begin{array}{c}\text { Sensitivity } \\
\mathbf{( 9 5 \% ~ C l )}\end{array}$ & $\begin{array}{c}\text { Specificity } \\
\mathbf{( 9 5 \% ~ C l )}\end{array}$ & $\begin{array}{c}\text { Positve Predictive } \\
\text { Value (95\% Cl) }\end{array}$ & $\begin{array}{c}\text { Negative Predictive } \\
\text { Value (95\% Cl) }\end{array}$ & Accuracy \\
\hline 60 & $94(70-99)$ & $24(8-47)$ & $48(30-66)$ & $83(36-99)$ & 54 \\
70 & $94(70-99)$ & $52(30-74)$ & $60(38-78)$ & $92(62-99)$ & 70 \\
80 & $94(70-99)$ & $66(43-85)$ & $68(45-86)$ & $93(68-99)$ & 78 \\
90 & $88(62-98)$ & $76(53-91)$ & $74(49-90)$ & $89(66-98)$ & 81 \\
100 & $69(41-88)$ & $81(58-94)$ & $73(45-92)$ & $77(55-92)$ & 76 \\
110 & $56(30-80)$ & $81(58-94)$ & $69(39-90)$ & $71(49-87)$ & 70 \\
120 & $50(25-75)$ & $90(70-98)$ & $80(44-97)$ & $70(50-86)$ & 73 \\
\hline
\end{tabular}

Figure 3. Receiver operating characteristic curve for various cut-offs of malondialdehyde-modified low-density lipoprotein (MDA$\mathrm{LDL}$ ) to differentiate between coronary artery disease with and without highly intense yellow plaque. Cl, confidence interval.

CAD. Serum MDA-LDL level may be a useful marker for the identification of vulnerable plaques.

Holvoet et al reported activated platelets that were associated with plaque instability to produce large amounts of aldehydes, further enhancing the modification of LDL; therefore circulating MDA-LDL was significantly elevated in patients with ACS. 4,5 The present findings are in line with the previous studies. Significant differences were seen in the serum levels of MDA-LDL between patients with and without ACS (127.7 \pm 66.4 U/L vs. 87.4 $\pm 33.1 \mathrm{U} / \mathrm{L}, \mathrm{P}=0.016)$. Furthermore, the complex plaques included coronary thrombus (except in 1 patient) and they were, although not statistically independent, associated with the serum level of MDA-LDL. These findings might partially support the mechanism of MAD-LDL elevation: that activated platelets generate large amounts of aldehydes, which cause the elevation of serum MAD-LDL. It would be, how- ever, difficult to explain why $44 \%$ of the present patients with elevated serum MDA-LDL (8/18) had neither ACS nor complex plaques including coronary thrombus, by this mechanism alone. A total of $63 \%$ of such patients with elevated MDALDL (5/8) were observed to have HIYP in this study. Furthermore, the present study found serum MDA-LDL level to be significantly correlated with yellow intensity at the plaques in CAD patients, and HIYP to be the only independent predictor of the elevation of serum MAD-LDL in patients with CAD. Therefore, we propose that the elevation of serum MAD-LDL is caused by the presence of HIYP.

Why was the yellow intensity of plaques correlated with serum MDA-LDL? The yellow intensity of plaques evaluated on angioscopy has been demonstrated to have an inverse relationship with plaque fibrous cap thickness in postmortem studies, ${ }^{7}$ in vitro, ${ }^{16}$ and in vivo using optical coherence tomography. ${ }^{9}$ 


\begin{tabular}{|c|c|c|c|}
\hline & MDA-LDL $<90 \mathrm{U} / \mathrm{L}(\mathrm{n}=19)$ & MDA-LDL >90 U/L $(n=18)$ & $P$ value \\
\hline Age (years) & $59.0 \pm 10.9$ & $61.3 \pm 7.4$ & 0.83 \\
\hline Male & $17(89)$ & $17(94)$ & $>0.99$ \\
\hline \multicolumn{4}{|l|}{ Risk factors } \\
\hline Diabetes mellitus & $6(32)$ & $5(28)$ & $>0.99$ \\
\hline Hypertension & $12(63)$ & 7 (39) & 0.24 \\
\hline Hyperlipidemia & $13(68)$ & $14(78)$ & 0.71 \\
\hline Smoking & $1(5)$ & $2(11)$ & $>0.99$ \\
\hline $\mathrm{BMI}\left(\mathrm{kg} / \mathrm{m}^{2}\right)$ & $24.6 \pm 3.9$ & $25.9 \pm 2.0$ & 0.60 \\
\hline \multicolumn{4}{|l|}{ Diagnosis } \\
\hline ACS & $2(11)$ & $8(44)$ & 0.03 \\
\hline AMI & $1(5)$ & 7 (39) & \\
\hline Unstable angina & $1(5)$ & $1(6)$ & \\
\hline Stable angina & $17(89)$ & $10(56)$ & \\
\hline \multicolumn{4}{|l|}{ Location of target lesion } \\
\hline $\mathrm{RCA}$ & $4(21)$ & $4(22)$ & 0.71 \\
\hline LAD & $11(58)$ & $12(67)$ & \\
\hline LCx & $4(21)$ & $2(11)$ & \\
\hline \multicolumn{4}{|l|}{ Serum lipid } \\
\hline MDA-LDL (U/L) & $65.9 \pm 13.2$ & $132.6 \pm 43.5$ & - \\
\hline Total cholesterol $(\mathrm{mg} / \mathrm{dl})$ & $181.2 \pm 27.5$ & $197.2 \pm 30.0$ & 0.10 \\
\hline $\mathrm{HDL}-\mathrm{C}(\mathrm{mg} / \mathrm{dl})$ & $49.1 \pm 16.8$ & $44.7 \pm 14.1$ & 0.40 \\
\hline Triglycerides (mg/dl) & $135.4 \pm 68.2$ & $163.6 \pm 68.4$ & 0.22 \\
\hline LDL-C (mg/dl) & $105.0 \pm 23.2$ & $119.7 \pm 25.5$ & 0.07 \\
\hline Statin use & $11(58)$ & $8(44)$ & 0.62 \\
\hline \multicolumn{4}{|l|}{ Angioscopic findings } \\
\hline HIYP & $2(11)$ & $14(78)$ & $<0.0001$ \\
\hline$b^{*}$ & $9.6 \pm 6.5$ & $25.9 \pm 7.3$ & $<0.0001$ \\
\hline Complex plaque & $3(16)$ & $9(50)$ & 0.03 \\
\hline
\end{tabular}

Data given as mean \pm SD or $n(\%)$

Abbreviations as in Table 1.

\begin{tabular}{|c|c|c|c|c|c|c|}
\hline & \multicolumn{3}{|c|}{ Univariate analysis } & \multicolumn{3}{|c|}{ Multivariate analysis } \\
\hline & $P$ value & OR & $95 \% \mathrm{Cl}$ & $P$ value & OR & $95 \% \mathrm{Cl}$ \\
\hline HIYP & $0.0003^{\dagger}$ & 29.75 & $4.72-187.14$ & $0.002^{\dagger}$ & 21.83 & $3.14-152.06$ \\
\hline Diagnosis of ACS & $0.030^{\dagger}$ & 6.80 & $1.19-38.56$ & 0.622 & 1.90 & $0.15-24.74$ \\
\hline Complex plaque & $0.033^{\dagger}$ & 5.33 & $1.14-24.90$ & 0.778 & 1.41 & $0.13-14.98$ \\
\hline
\end{tabular}

Variables with $\mathrm{P}>0.05$ on univariate analysis are not presented. †Statistically significant.

Abbreviations as in Tables 1,2.

Moreover, HIYP diagnosed on quantitative colorimetry was reported to have lipid under a thin $(<100 \mu \mathrm{m})$ fibrous cap. ${ }^{10}$ Such a thin fibrous cap has been reported to have extracellular matrix proteins degraded by proteolytic enzymes such as neutrophil elastase, plasminogen activators, and matrix metalloproteinases secreted from such activated inflammatory cells as macrophages and neutrophils, ${ }^{17}$ and be characterized by infiltration of those activated inflammatory cells. ${ }^{18}$ During such a condition of inflammation, they have been also shown to secrete myeloperoxidase (MPO), which catalyzes the conversion of chloride and hydrogen peroxide to hypochlorous acid $(\mathrm{HOCl}) .{ }^{19} \mathrm{HOCl}$ is considered to be implicated in the oxidation of plasma lipids contained within LDL-C, because it is easy for $\mathrm{HOCl}$ to leak from the degraded, loose fibrous cap. In fact, MPO has been reported to serve as a major enzymatic catalyst for initiation of lipid peroxidation in plasma. ${ }^{20}$

\section{Clinical Implications}

This study suggests that serum MDA-LDL level is a useful maker for the detection of vulnerable CAD patients before plaque disruption. Statin therapy has been reported to be effective in lowering both the yellow intensity of plaques ${ }^{21}$ and serum MDA-LDL level. ${ }^{22}$ The present data, however, could indicate that moderate statin therapy is insufficient for plaque stabilization, because the frequency of statin use with standard doses was not significantly associated with the elevation of serum MAD-LDL in the present study. We therefore consider that patients with elevated MDA-LDL need more intensive statin therapy. ${ }^{23}$ Serum MDA-LDL level may enable us to identify patients who may particularly benefit from more intensive statin therapy, although further studies are needed to address this important issue. 


\section{Study Limitations}

The present findings were based on observations in a relatively limited number of patients, and excluded patients with multiple vessel disease. We could not evaluate systemic arteries except the coronary artery. Therefore, the data may neglect the effect of other atherosclerotic lesions on serum MDA-LDL level. This may be the reason why the positive predictive value of MDA-LDL for HIYP is relatively low in comparison with the negative predictive value. In the present study, the yellow intensity $\left(b^{*}\right)$ on quantitative colorimetry was not examined on pathohistology, but we used the same colorimetry system as the previous pathohistological study with regard to $b^{*},{ }^{10}$ and the AMI patients were found to have a higher prevalence of HIYP diagnosed according to $b^{*}$ than those with stable angina ( $87 \%$ vs. $30 \%, \mathrm{P}=0.009$ ). These findings, including the frequency of HIYP, were in line with those of previous studies using $b^{*} .11,12$

\section{Conclusions}

Serum MDA-LDL level has a close correlation with the yellow intensity of plaque. Serum MDA-LDL level was a useful marker for the identification of thin-cap atheroma, using angioscopy and quantitative colorimetry. The present findings therefore confirm the hypothesis that elevated MDA-LDL reflects plaque instability in patients with CAD.

\section{Disclosures}

No financial support and no conflict of interest.

\section{References}

1. Brown MS, Goldstein JL. Lipoprotein metabolism in the macrophage: Implications for cholesterol deposition in atherosclerosis. Annu Rev Biochem 1983; 52: 223-261.

2. Steinberg D. Role of oxidized LDL and antioxidants in atherosclerosis. Adv Exp Med Biol 1995; 369: 39-48.

3. Steinberg D, Lewis A. Conner memorial lecture: Oxidative modification of LDL and atherogenesis. Circulation 1997; 95: 1062-1071.

4. Holvoet P, Vanhaecke J, Janssens S, Van de Werf F, Collen D. Oxidized LDL and malondialdehyde modified LDL in patients with acute coronary syndromes and stable coronary artery disease. Circulation 1998; 98: $1487-1494$.

5. Holvoet P, Collen D, Van de Werf F. Malondialdehyde-modified LDL as a marker of acute coronary syndromes. JAMA 1999; 281: $1718-1721$.

6. Mizuno K, Miyamoto A, Satomura K, Kurita A, Arai T, Sakurada M, et al. Angioscopic coronary macromorphology in patients with acute coronary disorders. Lancet 1991; 337: 809-812.

7. Uchida Y, Nakamura F, Tomaru T, Morita T, Oshima T, Sasaki T, et al. Prediction of acute coronary syndromes by percutaneous coronary angioscopy in patients with stable angina. Am Heart J 1995; 130: 195-203.

8. Ueda Y, Ohtani T, Shimizu M, Hirayama A, Kodama K. Assessment of plaque vulnerability by angioscopic classification of plaque color. Am Heart J 2004; 148: 333-335.

9. Takano M, Jang IK, Inami S, Yamamoto M, Murakami D, Okamatsu $\mathrm{K}$, et al. In vivo comparison of optical coherence tomography and angioscopy for the evaluation of coronary plaque characteristics. Am J Cardiol 2008; 101: 471-476.

10. Ishibashi F, Yokoyama S, Miyahara K, Dabreo A, Weiss ER, Iafrati M, et al. Quantitative colorimetry of atherosclerotic plaque using the $\mathrm{L} * \mathrm{a} * \mathrm{~b}$ color space during angioscopy for the detection of lipid cores underneath thin fibrous cap. Int J Cardiovasc Imaging 2007; 23: 679-691.

11. Ishibashi F, Mizuno K, Kawamura A, Singh PP, Nesto RW, Waxman S. High yellow color intensity by angioscopy with quantitative colorimetry to identify high-risk features in culprit lesions of patients with acute coronary syndrome. Am J Cardiol 2007; 100: 1207-1211.

12. Inami S, Ishibashi F, Waxman S, Okamatsu K, Seimiya K, Takano M, et al. Multiple yellow plaques assessed by angioscopy with quantitative colorimetry in patients with myocardial infarction. Circ J 2008; 72: $399-403$.

13. Yamamoto M, Takano M, Okamatsu K, Murakami D, Inami S, Xie Y, et al. Relationship between thin cap fibroatheroma identified by virtual histology and angioscopic yellow plaque in quantitative analysis with colorimetry. Circ J 2009; 73: 497-502.

14. Kotani K, Maekawa M, Kanno T, Kondo A, Toda N, Manabe M. Distribution of immunoreactive malondialdehyde-modified low-density lipoprotein in human serum. Biochim Biophys Acta 1994; 1215: $121-125$.

15. Okamatsu K, Takano M, Sakai S, Ishibashi F, Uemura R, Takano T, et al. Elevated troponin T levels and lesion characteristics in non-STelevation acute coronary syndromes. Circulation 2004; 109: $465-$ 470.

16. Miyamoto A, Prieto AR, Friedl SE, Lin FC, Muller JE, Nesto RW, et al. Atheromatous plaque cap thickness can be determined by quantitative color analysis during angioscopy: Implications for identifying the vulnerable plaque. Clin Cardiol 2004; 27: 9-15.

17. Kobayashi N, Hata N, Kume N, Yokoyama S, Shinada T, Tomita K, et al. Matrix metalloproteinase- 9 for the earliest stage acute coronary syndrome. Circ J 2011; 75: 2853-2861.

18. Tavora FR, Ripple M, Li L, Burke AP. Monocytes and neutrophils expressing myeloperoxidase occur in fibrous caps and thrombi in unstable coronary plaques. BMC Cardiovasc Disord 2009; 9: 27.

19. Loria V, Dato I, Graziani F, Biasucci LM. Myeloperoxidase: A new biomarker of inflammation in ischemic heart disease and acute coronary syndromes. Mediators Inflamm 2008; 2008: 135625.

20. Zhang R, Shen Z, Nauseef WM, Hazen SL. Defects in leukocytemediated initiation of lipid peroxidation in plasma as studied in myeloperoxidase-deficient subjects: Systematic identification of multiple endogenous diffusible substrates for myeloperoxidase in plasma. Blood 2002; 99: 1802-1810.

21. Takano M, Mizuno K. Coronary angioscopic evaluation for serial changes of luminal appearance after pharmacological and catheter interventions. Circ J 2010; 74: 240-245.

22. Tani S, Watanabe I, Anazawa T, Kawamata H, Tachibana E, Furukawa $\mathrm{K}$, et al; Surugadai Atherosclerosis Regression Investigators. Effect of pravastatin on malondialdehyde-modified low-density lipoprotein levels and coronary plaque regression as determined by three-dimensional intravascular ultrasound. Am J Cardiol 2005; 96: 1089-1094.

23. Cholesterol Treatment Trialists' (CTT) Collaboration. Efficacy and safety of more intensive lowering of LDL cholesterol: A meta-analysis of data from 170,000 participants in 26 randomised trials. Lancet 2010; 376: $1670-1681$. 